Éducation et francophonie

\title{
Le constructivisme en didactique des sciences
}

Dilemmes et défi

Constructivism in science education

Dilemmas and challenges

\section{El constructivismo en didáctica de las ciencias}

\section{Dilemas y desafíos}

\section{Bernard Laplante}

Volume 25, numéro 1, printemps 1997

L'apprentissage et l'enseignement des sciences et des mathématiques dans une perspective constructiviste

URI : https://id.erudit.org/iderudit/1080655ar

DOI : https://doi.org/10.7202/1080655ar

Aller au sommaire du numéro

Éditeur(s)

Association canadienne d'éducation de langue française

ISSN

0849-1089 (imprimé)

1916-8659 (numérique)

Découvrir la revue

Citer cet article

Laplante, B. (1997). Le constructivisme en didactique des sciences : dilemmes et défi. Éducation et francophonie, 25(1), 166-182.

https://doi.org/10.7202/1080655ar
Résumé de l'article

Depuis une vingtaine d'années, le constructivisme en didactique des sciences semble être à la mode. Ainsi, à la suite des recherches portant sur les conceptions des élèves, de nombreux modèles pédagogiques d'orientation constructiviste ont été proposés. Pourtant ces modèles n'arrivent pas à s'imposer. Certains obstacles semblent freiner, voire même empêcher, l'intégration d'une perspective constructiviste à l'enseignement des sciences. Cet article explore certains aspects de cette problématique afin de cerner la nature des dilemmes et des défis auxquels les tenants d'une perspective constructiviste ont à faire face.

Tous droits réservés @ Association canadienne d'éducation de langue française, Ce document est protégé par la loi sur le droit d’auteur. L’utilisation des 1997 services d’Érudit (y compris la reproduction) est assujettie à sa politique d'utilisation que vous pouvez consulter en ligne.

https://apropos.erudit.org/fr/usagers/politique-dutilisation/ 


\section{Le constructivisme en didactique des sciences Dilemmes et défi}

\section{Bernard LAPLANTE}

Université de Regina, Saskatchewan, Canada

\section{RÉSUMÉ}

Depuis une vingtaine d'années, le constructivisme en didactique des sciences semble être à la mode. Ainsi, à la suite des recherches portant sur les conceptions des élèves, de nombreux modèles pédagogiques d'orientation constructiviste ont été proposés. Pourtant ces modèles n'arrivent pas à s'imposer. Certains obstacles semblent freiner, voire même empêcher, l'intégration d'une perspective constructiviste à l'enseignement des sciences. Cet article explore certains aspects de cette problématique afin de cerner la nature des dilemmes et des défis auxquels les tenants d'une perspective constructiviste ont à faire face. 


\section{ABSTRACT}

\section{Constructivism in science education - dilemmas and challenges}

Bernard LAPLANTE

University of Regina, Saskatchewan, Canada

For over twenty years, constructivism has been prominent in science education. As the result of an extensive research program on students' conceptions, various constructivist oriented teaching models have been developed. Nonetheless, it seems that a number of obstacles are delaying, if not preventing, the integration of these teaching models in the science classroom. This article explores some of the dilemmas and challenges facing supporters of a constructivist perspective in science education.

\section{RESUMEN}

\section{El constructivismo en didáctica de las ciencias - dilemas y desafíos}

Bernard LAPLANTE

Universidad de Regina, Saskatchewan, Canadá

Desde hace unos veinte años se puso a la moda el constructivismo en la didáctica de las ciencias. Las investigaciones sobre las concepciones de los alumnos inspiraron la proposición de un sinnúmero de modelos pedagógicos de orientación constructivista Sin embargo, dichos modelos no han logrado imponerse. Algunos obstáculos parecen frenar e incluso impedir la integración de una perspectiva constructivista en la enseñanza de las ciencias. Este artículo explora algunos aspectos de dicha problemática con el objeto de circunscribir el tipo de dilemas y desafíos que tienen que confrontar los promotores de la perspectiva constructivista.

\section{Introduction}

Depuis une vingtaine d'années, le constructivisme est à la mode. C'est ainsi que les recherches portant sur les conceptions des élèves à propos des objets et des phénomènes qui les entourent sont devenues le moteur de presque tous les efforts visant à améliorer l'enseignement et l'apprentissage des sciences à l'école (Treagust, Duit et Fraser, 1996). À la suite de ces recherches, de nombreux modèles pédagogiques ont été proposés dans le but d'amener les élèves à changer ou complexifier leurs conceptions. Pourtant, malgré leur attrait certain, ces modèles n'arrivent pas à s'imposer (Duit, 
1995; Larochelle et Bednarz, 1994). Certains obstacles semblent freiner, voire empêcher, l'intégration d'une perspective constructiviste à l'enseignement des sciences.

Il faut dire que l'épistémologie constructiviste place les différents acteurs ${ }^{1}$ qui oeuvrent en didactique des sciences devant de nombreux dilemmes. Selon les choix pour lesquels ils optent - rester dans leurs habitudes ou changer et apprendre -, ces acteurs peuvent avoir à relever des défis de taille. Cet article explore certains aspects de cette problématique afin de cerner la nature des dilemmes et des défis auxquels les tenants d'une perspective constructiviste ont à faire face.

Pour bien se situer dans le contexte de la classe et illustrer certaines caractéristiques de l'épistémologie constructiviste, nous discutons d'abord de la nature des conceptions des élèves et du rôle qu celles-ci jouent dans l'apprentissage des sciences. Puis nous présentons les grandes lignes de certains modèles pédagogiques d'orientation constructiviste afin d'illustrer leur complexité et le défi que pose leur mise en oeuvre en milieu scolaire. Ensuite, nous discutons du cas de deux enseignantes de première année pour mieux comprendre ce qui, à notre sens, constitue un des obstacles majeurs à l'intégration de la thèse constructiviste à l'enseignement des sciences au primaire. Enfin, nous concluons en apportant des suggestions qui pourraient faciliter une telle intégration.

\section{Les conceptions des élèves en sciences}

Comme le souligne Désautels (1983), les enfants n'attendent pas d'aller à l'école pour formuler des explications aux phénomènes naturels dont ils font l'expérience. Ainsi, avant même d'avoir suivi des cours de sciences, les enfants ont élaboré de nombreuses conceptions à propos de notions aussi variées que l'origine des roches, l'énergie, la photosynthèse, l'électricité et le comportement des animaux. Ces conceptions correspondent aux connaissances personnelles des élèves par rapport à un phénomène quelconque; elles marquent également leur façon de penser et de raisonner face à des situations qui y font référence. Il faut donc voir ces conceptions en tant que produits et outils de la pensée. Ces conceptions sont souvent différentes de celles généralement acceptées par les scientifiques. De plus, elles sont tenaces et elles résistent aux efforts qui sont faits pour les amener à évoluer.

À titre d'exemple, considérons d'abord le cas de cette fillette de 6 ans qui a dessiné sa chienne qui attend un petit (voir la figure plus bas). En interagissant avec son environnement et surtout en interrogeant les gens qui l'entourent, si l'on peut en juger d'après son dessin, elle en est arrivée à élaborer un premier niveau de formulation des conceptions pour le moins intéressantes de la gestation, de la digestion et de la pensée chez les animaux. Ces conceptions forment un savoir pratique auquel elle fera sûrement appel pour donner un sens à des expressions comme: «Il faut que

1. Ces acteurs comprennent les personnes œuvrant directement dans la salle de classe (élèves, enseignants et enseignantes), celles engagées dans la recherche, mais aussi et, de leur propre façon, puisqu'ils ne sont pas indéfiniment malléables et parlent au nom de ceux et celles qui les ont conçus, les programmes d'études en sciences et les ressources pédagogiques utilisées en classe. 
maman mange pour nourrir le bébé qui est dans son ventre.» Cette fillette fera également référence à ces conceptions pour répondre à des questions portant sur la pensée des animaux.

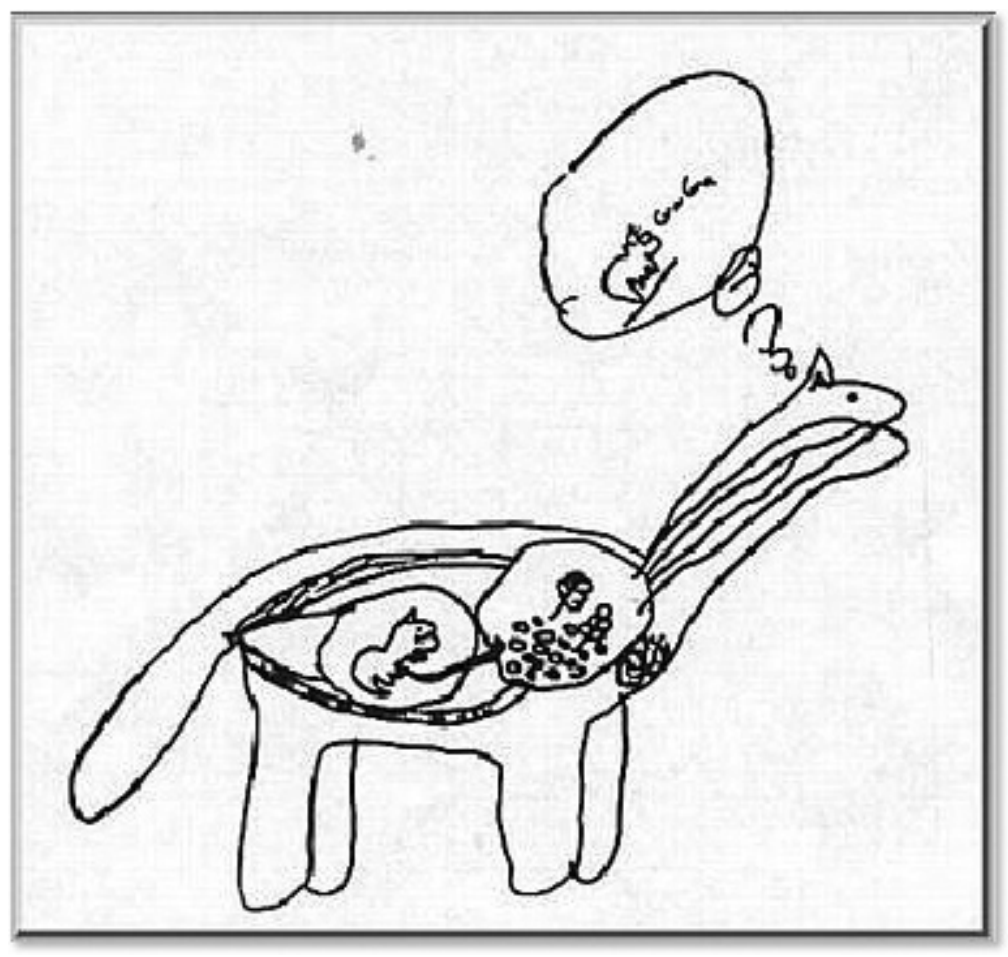

Chienne qui attend un petit (collection de l'auteur: 1986).Le fœetus flotte dans l'utérus. Il est nourri par un cordon directement relié à l'estomac de la mère. Cette dernière semble savoir qu'elle attend un petit; elle pense à son bébé qui dit «GaGa».

Considérons également le cas de cette élève de troisième année à qui l'enseignant demandait ce qui arrive quand on met du sel dans l'eau. Alors que plusieurs élèves ont répondu: «Le sel fond», elle lui a dit: «Le sel va flotter à la surface de l'eau [...] l'été dernier je suis allée nager à la mer et le dessus de l'eau goûtait salé» (Entendu par l'auteur, novembre, 1996).

Ces deux exemples illustrent à leur façon que les expériences vécues sont à la source des conceptions et que c'est à partir de ces mêmes conceptions que les apprenants parviennent à donner un sens à de nouvelles expériences. La cognition est active tout au long de ce processus de construction de sens qui demeure individuel et idiosyncratique. Comme le dit si habilement Duit (1995), «Les conceptions guident les perceptions qui à leur tour développent les conceptions» (p. 280; traduction libre). De la même façon, les apprenants ne reçoivent pas passivement les connaissances qui leur sont présentées. Ils ne les comprennent qu'en construisant un sens à partir de leurs propres conceptions. Aux yeux des apprenants, les conceptions sont fort utiles et se montrent viables, non pas parce qu'elles correspondraient à la réalité des choses telles qu'elles sont, mais dans la mesure où elles leur permettent 
d'organiser le monde des expériences et de résoudre les problèmes auxquels ils ont à faire face (Ruel, 1992) 2 .

Ces conceptions représentent un dilemme important pour les praticiens de l'enseignement des sciences. Doit-on continuer à les ignorer (avec les résultats qu'on connaît)? Sinon, comment doit-on les intégrer à l'enseignement? De nombreux chercheurs et chercheuses se sont penchés sur cette question. Étant donné la nature de ces conceptions et le rôle qu'elles jouent dans l'apprentissage, il est peu surprenant que, depuis une vingtaine d'années, leur étude soit devenue le moteur de presque tous les efforts visant à améliorer l'enseignement et l'apprentissage en sciences (Treagust, Duit et Fraser, 1996). Des centaines d'articles publiés sur le sujet en répertorient les multiples variations ${ }^{3}$. De nombreux livres explorent le rôle que ces conceptions jouent dans l'apprentissage ${ }^{4}$. Ces travaux «ont contribué à une meilleure compréhension de la formation des connaissances par l'apprenant et à l'élaboration de situations didactiques susceptibles de favoriser leur évolution" (Larochelle et Bednarz, 1994, p. 5).

\section{Modèles pédagogiques d'orientation constructiviste}

Devant de telles conceptions, qui font souvent obstacle à l'apprentissage, les chercheurs et les chercheuses en didactique ont développé de nombreux modèles pédagogiques qui cherchent à changer, faire évoluer ou encore complexifier ces conceptions. La plupart de ces modèles se fondent essentiellement sur la même approche. Ils cherchent d'abord à mettre au jour les conceptions des élèves, à amener ces derniers à les décrire et à les expliquer ouvertement en classe, puis, au moyen d'activités d'exploration et d'investigation, à amener les élèves à mettre leurs conceptions à l'épreuve tout en leur présentant un point de vue scientifique et enfin, au moyen d'autres activités, à amener les élèves à explorer cette nouvelle conception afin de mieux la comprendre et d'en voir les avantages (Driver, 1983).

Ainsi en est-il du modèle de changement conceptuel avancé par Hewson (1981) et Nussbaum et Novick (1982). Ce modèle demande d'abord que l'enseignant mette au jour les conceptions des élèves, puis crée une situation didactique dans laquelle ils n'arrivent pas à expliquer un «incident critique». Le but poursuivi ici est de susciter chez eux un conflit conceptuel qui ne pourra voir sa résolution que dans l'accommodation de leurs conceptions initiales à la lumière de la conception appropriée présentée par l'enseignant. Bien sûr, l'incident critique proposé par l'enseignant n'est pas nécessairement perçu comme tel par les élèves, qui ne sont alors pas prêts

2. Les deux postulats de l'épistémologie constructiviste se résument donc à ceci: les connaissances sont activement construites par le sujet connaissant et elles ne sont validées que dans la mesure où elles se montrent viables, la fonction de la cognition étant essentiellement adaptative (Ruel, 1992).

3. Voir entre autres ceux cités par Fensham, Gunstone et White (1994), Gallas (1995), Giordan, Girault et Clément (1994), Larochelle et Désautels (1992), Treagust, Duit et Fraser (1996) et Wandersee, Mintzes et Novack (1994).

4. En plus des livres cités à la note précédente, voir Astolfi et Devalay (1989), Driver (1983), Driver, Guesne et Tiberghien (1985), Fosnot (1996), Giordan (1983), Steffe et Gale (1995) et Tobin (1993). 
à simplement rejeter leurs conceptions initiales. Les élèves, tout comme les scientifiques, ne changent pas d'idée uniquement pour des raisons rationnelles (Cleminson, 1990).

Strike et Posner (1982) et par la suite Hewson et Hewson (1984) ont précisé les conditions les plus favorables au changement conceptuel ${ }^{5}$. Il faudrait d'abord que les élèves éprouvent un certain degré d'insatisfaction à l'égard de la conception initiale. Ensuite, il faudrait que la nouvelle conception qu'on leur présente soit à leurs yeux intelligible, plausible et féconde. Ces conditions affectent directement le statut que différentes conceptions peuvent avoir aux yeux des élèves. Dans un tel contexte, le rôle de l'enseignant est de mettre en place des conditions qui permettent de modifier le statut des conceptions en jeu, par exemple en montrant que les conceptions initiales ne peuvent pas expliquer certains phénomènes, alors que d'autres le peuvent, tout en s'assurant que les nouvelles conceptions remplissent les autres conditions mentionnées plus haut. Le travail sur le plan métacognitif devient très important, alors que l'enseignant, après avoir mis au jour les conceptions initiales des élèves et présenté un point de vue scientifique, amène ces derniers à discuter ouvertement du pour et du contre des différentes conceptions présentées. Bien sûr, une telle approche implique des changements importants chez tous les participants quant à leur conception du rôle et des responsabilités de l'enseignant et des élèves. Elle demande également qu'un climat de confiance règne dans la classe de façon que les conceptions tant de l'enseignant que des élèves puissent être remises en question et évaluées.

Une critique souvent formulée à l'égard du modèle de changement conceptuel est qu'il déprécie aux yeux mêmes des apprenants des conceptions qui jusqu'alors se sont montrées utiles et viables. Ce qu'il faudrait plutôt arriver à leur faire réaliser, c'est que le contexte dans lequel les connaissances scientifiques s'appliquent n'est pas le même que celui du savoir commun. Comme l'expliquent Larochelle et Désautels (1992), il s'agit de deux jeux de la connaissance différents. Il ne faut pas en arriver à juger les connaissances d'un jeu à partir des règles de l'autre. Il faudrait plutôt chercher à rendre les élèves conscients des postulats épistémologiques et des croyances métaphysiques qui contrôlent l'élaboration du savoir scientifique afin qu'ils puissent jouer le jeu de la science en toute connaissance de cause.

D'autres chercheurs, dont Bednarz et Garnier (1989), De Vecchi et Giordan (1989) et Astolfi et Peterfalvi (1993), ont repris la notion de conflit en insistant sur l'importance de susciter un conflit à caractère sociocognitif chez tous les élèves de la classe. Ils ont également introduit l'idée d'obstacle épistémologique qui représenterait le noyau dur de certaines conceptions (Astolfi et Peterfalvi, 1993). Un exemple d'obstacle est la primauté que certains élèves donnent à la perception sur la conceptualisation en tant que source d'explication (la chaleur perçue comme sensation plutôt que comme concept). Ce serait justement sur ces obstacles épistémologiques

5. Il ne faut pas comprendre cette notion au sens strict de terme changement. En ce sens, Hewson (1996) a précisé qu'un tel changement ne se limitait pas nécessairement au remplacement d'une conception par une autre, mais pouvait aussi inclure l'adoption d'une nouvelle conception, la restructuration d'une conception déjà existante, ou, dans certains cas, la coexistence de deux conceptions. 
que devrait porter tout le travail didactique. Selon le modèle proposé, il faudrait amener les élèves à prendre conscience de la conception qui fait obstacle, puis susciter un conflit sociocognitif dans le but de fissurer l'obstacle en question pour, ensuite, le franchir en proposant aux élèves un «modèle explicatif alternatif» le remplaçant (Astolfi et Peterfalvi, 1993, p. 118). Enfin, cette phase de reconstruction serait suivie d'une période de consolidation et d'automatisation de ce nouveau «modèle». Bien sûr, une telle approche pédagogique n'est pas sans causer de difficultés. Ainsi, les élèves peuvent ne pas percevoir comme contradictoires certaines situations qui leur sont présentées ou ils peuvent donner trop vite «la bonne réponse» afin d'éviter un véritable travail sur l'obstacle. Certains élèves peuvent tout simplement se sentir découragés et dévalorisés par rapport au travail à accomplir (Astolfi et Peterfalvi, 1993). De plus, les situations d'enseignement et d'apprentissage qui font appel aux notions de conflit sociocognitif et d'obstacle épistémologique sont très exigeantes pour ceux et celles qui y participent, ne serait-ce qu'en raison du travail sur les plans épistémologique et affectif qu'elles supposent.

D'autres modèles pédagogiques d'orientation constructiviste ont également été développés. On pense entre autres au modèle allostérique (De Vecchi et Giordan, 1989), à celui du dérangement épistémologique de Larochelle et Désautels (1992) et à ceux liés aux projets PEEL et TLSS où la métacognition occupe une place importante (Baird et White, 1996). Ce qui est remarquable à propos de plusieurs de ces modèles, c'est leur complexité et le défi que pose leur mise en œuvre en milieu scolaire. Bien sûr, ils ont été mis à l'essai en classe, mais souvent dans des situations assez différentes de celles rencontrées dans des classes du primaire où, entre autres, les enseignants et les enseignantes généralistes travaillent avec des groupes d'élèves de plus en plus nombreux. C'est en partie ce qui a amené des chercheurs à remettre en question la faisabilité didactique de certains de ces modèles pédagogiques (Arcà et Caravita, 1993; Astolfi et Peterfalvi, 1993). Comme le souligne Duit (1995), les stratégies d'orientation constructiviste connaissent des succès limités. De plus, elles ne rendent pas l'enseignement et l'apprentissage des sciences plus aisés; au contraire, elles créent même des situations plus exigeantes pour les acteurs qui s'y engagent.

\section{Attrait de la perspective constructiviste et intégration aux pratiques éducatives}

De prime abord, la perspective constructiviste paraît fort attrayante. Ainsi, les modèles pédagogiques qui adoptent une telle perspective proposent généralement des activités d'apprentissage en situation qui savent intéresser les élèves au plus haut point. Il s'agit d'activités authentiques et significatives qui placent les élèves au centre de leur apprentissage en mobilisant leurs connaissances antérieures tant sur le plan des savoirs qu'au regard des savoir-faire et des savoir-être. Certains modèles, par la réflexion métacognitive qu'ils encouragent chez les élèves, les initient à des concepts relatifs à la nature des connaissances scientifiques et de leurs modes de production, tout en leur permettant de prendre conscience de leur propre mode de pensée. Enfin, 
ces modèles amènent des changements importants dans la façon dont les choses se déroulent en classe en favorisant une négociation plus grande des processus et des contenus d'apprentissage. Il s'agit donc de modèles qui mènent à l'élaboration d'un contexte d'apprentissage très riche et émancipatoire pour ceux et celles qui y participent, qui changent non seulement le rapport au savoir, mais également le rapport au pouvoir.

Cependant, les modèles pédagogiques qui adoptent une perspective constructiviste n'arrivent pas à s'imposer. Comme le suggèrent Larochelle et Bednarz (1994), «l'intégration de la thèse constructiviste au renouvellement des pratiques éducatives demeure marginale» (p. 5). Pour les tenants de la thèse constructiviste, il s'agit à la fois d'un dilemme et d'un véritable défi. Comment s'expliquer un tel état de choses? Se pourrait-il que, tenant compte du contexte d'enseignement des sciences au primaire, les enseignants et les enseignantes aient développé un savoir pratique viable qui aurait assez peu en commun avec l'orientation constructiviste? En ce sens, quelles sont les stratégies pédagogiques mises en œuvre durant leurs cours de sciences? Quels en sont les fondements épistémologiques? En d'autres mots, comment ces enseignantes et ces enseignants se représentent-ils l'enseignement et l'apprentissage des sciences?

\section{Cas de deux enseignantes du primaire en immersion française}

Deux enseignantes du primaire observées lors d'une récente étude permettent d'apporter certains éléments de réponse à ces questions (Laplante, 1993, 1996,1997) . Nous avons entrepris une étude dans le but d'établir des liens, d'une part entre les conceptions de la langue et de la science que se font des enseignantes et, d'autre part, les stratégies pédagogiques observées dans leur classe. En raison de la nature des questions de recherche, nous avons choisi de travailler dans la salle de classe, de favoriser des modes qualitatifs de collecte de données, d'analyser les données de façon inductive, de négocier avec les participantes nos interprétations et de présenter nos résultats sous forme d'étude de cas. Nous avons observé, pendant plus de 40 heures, deux enseignantes de première année en immersion française durant leurs cours de sciences ${ }^{6}$. Ces observations se sont étalées sur une période de douze mois. Elles se sont déroulées dans deux écoles d'une grande ville des Prairies offrant un enseignement régulier en anglais et un programme d'immersion longue $\left(\mathrm{M}-8^{\mathrm{e}}\right)$ à plus de 400 élèves. Durant ces observations, les conversations entre les enseignantes et leurs élèves ont été enregistrées, pour ensuite être retranscrites et analysées de façon à décrire les stratégies pédagogiques mises en œuvre. Dans le but d'explorer les conceptions des enseignantes, nous les avons rencontrées lors d'entretiens semi-structurés

6. Les programmes d'immersion française offrent la possibilité à des élèves anglophones de suivre, dès la maternelle, tous leurs cours en français, leur permettant ainsi de développer une compétence de communication de cette langue. 
menés selon un protocole comportant des questions sur la nature de la langue, sur celle de la science et sur l'élève en tant qu'apprenant. Nous avons retranscrit ces entretiens qui ont fait l'objet d'une analyse de nature descriptive et interprétative. Enfin, nous avons tracé les liens entre les stratégies pédagogiques observées et les conceptions des enseignantes.

Les deux enseignantes observées donnaient un enseignement fondé sur l'exploitation de grands thèmes tirés du programme de sciences, un enseignement dont le déroulement était contrôlé de près et dans lequel le "pourquoi » et le «comment" des activités faisaient rarement l'objet d'une discussion, un enseignement comportant de nombreuses leçons formelles visant essentiellement la transmission des connaissances et quelques activités dites de découverte, un enseignement marqué par une façon particulière de présenter les objets d'études en sciences comme étant donnés, tenant de l'évidence même et de nature non problématique. Dans un tel contexte, les conceptions des élèves faisaient rarement surface et, quand cela se produisait, elles étaient souvent ignorées ou mises en doute. À vrai dire, les élèves étaient considérés comme de simples récepteurs de connaissances transmises par l'enseignante?

Nous allons illustrer ce que nous avançons ici au moyen de deux activités observées dans la classe d'une de ces enseignantes. La première activité porte sur le printemps; elle s'organise autour d'une feuille de travail illustrant des signes que certains associent au printemps (la neige qui fond, les oiseaux qui reviennent du sud, les bourgeons qui s'ouvrent, etc.). L'enseignante amorce la discussion et en contrôle le déroulement en demandant à plusieurs reprises aux élèves de nommer certains de ces signes, limitant ainsi ce dont ils peuvent parler. Malgré tout, tout au long des échanges, les idées des élèves font surface. Il arrive que l'enseignante les incorpore à la conversation, mais souvent des idées fort intéressantes sont complètement ignorées, comme l'illustre l'extrait suivant:

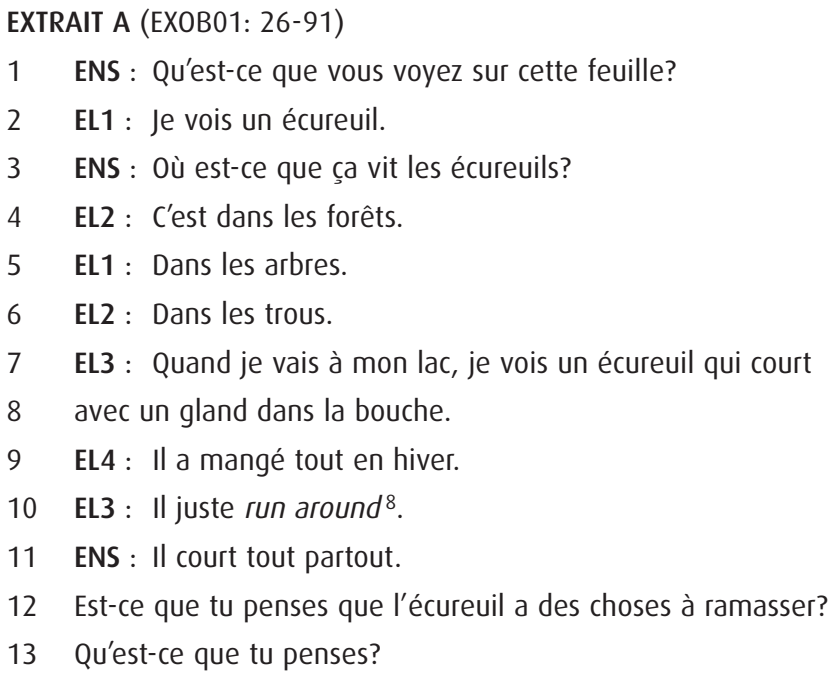

7. Ces idées sont développées plus à fond dans Laplante (1993, 1996, 1997).

8. Cette activité s'est déroulée dans une classe de première année en immersion française, ce qui explique que les élèves emploient certaines expressions anglaises. 
Cette conversation semble exploitée de façon assez intéressante du point de vue langagier. L'intervention d'un premier élève (EL1) est incorporée à la conversation alors que l'enseignante (ENS) lui demande de s'expliquer (2 et 3 ). D'autres élèves sont ainsi amenés à participer (4 à 9). Ensuite, l'enseignante traduit ce qu'un autre élève vient de dire en anglais (10 et 11), puis elle lui demande de s'expliquer (12-13). Quelques instants plus tard (14), elle relance la conversation, mais les élèves s'éloignent de l'idée de printemps (15 à 18). Deux élèves interviennent alors en suggérant des idées fort pertinentes, mais qui ne sont pas incorporées à la conversation (19 et 20). L'enseignante ramène la conversation sur le sujet des écureuils (21). Le but de cette activité semble être d'amener les élèves à discuter des signes annonçant le printemps tels qu'ils sont illustrés sur la feuille de travail et tels que l'enseignante se les représente, et ce, sans trop tenir compte de la conception que les élèves se font du printemps.

La seconde activité porte sur l'observation d'une graine de haricot. Elle s'est déroulée après que les élèves eurent lu l'histoire Jacques et le haricot magique. L'enseignante amorce l'activité en distribuant une graine à chacun des élèves tout en leur disant:

Ça, c'est une graine de haricot. Et vous allez la regarder de tous les côtés. Et puis, ensuite, qu'est-ce que vous allez faire? Regardez bien. Vous allez la briser avec votre ongle, comme ça, et vous allez l'ouvrir pour regarder ce qu'il y a dedans. Et moi, je m'aperçois de quelque chose, mais j'ai bien hâte de voir si vous êtes capables de vous apercevoir de quelque chose aussi (EXOB031292).

Bien que l'enseignante précise le «comment» («briser avec votre ongle») et le «pourquoi» («regarder ce qu'il y a dedans»), ces éléments essentiels de la démarche ne font pas l'objet d'une discussion. Le dernier commentaire peut laisser aux élèves l'impression que l'enseignante s'attend à ce qu'ils voient le même «quelque chose» qu'elle. En d'autres mots, il suffirait de regarder attentivement pour observer ce qu'il y a à observer. En ce sens, il aurait été intéressant d'amener les élèves à s'interroger sur leur conception d'une graine de façon à orienter leurs observations. Après une période d'observation d'une dizaine de minutes, l'enseignante lance une discussion dont elle contrôle le déroulement au moyen de questions. 
EXTRAIT B (EXOB03: 1486-1533 et 1662-1697)

1 ENS : Qu'est-ce que tu as vu? [à EL1]

2 EL1 : J'ai vu un petit spot brun/

3 ENS: Des petites quoi? ...taches.

4 EL1: Taches brun/ Et que je le split...

5 ENS : Quand j'ai... [l'enseignante fait le geste d'ouvrir]

6 EL1 : Ouvrir... J'ai vu une petite... une petite tige et des feuilles.

7 ENS : OK. D'accord... Bon, tu as dis que tu as regardé, et tu as

8 vu... euh... tu as vu des taches noires sur la plante?

9 EL1 : Oui...

10 ENS : Mais, quand tu as ouvert, qu'est-ce que tu as découvert qu'il y avait...

11 EL1 : Une plant.

12 EL2: Une petite plante.

13 ENS : Non, qu'est-ce qui avait en dehors? Qu'est-ce qui était là?

14 EL1 : Le couvercle.

15 ENS : C'est une quoi? C'est une belle petite couverture comme ça.

16 C'est comme de la peau...

17 ... [quelques instants plus tard]

18 ENS : Il y a une autre partie à la graine. Il y a le dedans, puis ensuite...

19 Qu'est-ce que c'est le dedans?

20 Qu'est-ce que ça fait cette partie en dedans de la peau de la graine?

21 EL3 : Il fait les haricots...

22 ENS : Qu'est ce que tu penses? [à EL2] Qu'est-ce qu'il y a dedans?

23 Ces deux parties, ici là. C'est quoi ça?

24 EL2 : Le... le [il hésite]

25 ENS : Est-ce que quelqu'un peut le dire?

26 EL4: Le graine... La graine.

27 ENS : C'est la partie d'une graine, oui.

28 Et puis qu'est-ce qu'on fait... Et qu'est ce qui arrive avec ça?

29 EL5 : On plante et ça grow.

30 ENS : On plante et ça...

31 EL6 : ... pousse.

32 ENS : Ça pousse, et puis on trouve la graine.

33 Qu'est-ce qui sort de cette graine? [à EL7]

34 EL7 : Le... le petite plante.

L'enseignante (ENS) lance la discussion en posant une question ouverte (1) à laquelle un élève (EL1) s'empresse de répondre (2). Elle l'aide à élaborer et enrichir son observation (3 à 8), mais elle ne semble pas satisfaite de cette observation, puisqu'à deux reprises elle pose des questions de plus en plus fermées (10 et 13). Finalement, elle suggère la réponse recherchée (15-16). Elle s'engage alors dans une longue discussion à propos de la fonction de la peau (17). Par la suite, elle poursuit son questionnement encore de façon fermée (18-20). Le discours de l'enseignante et celui des élèves se rejoignent à peine. Bien qu'ils fassent référence au même objet, ils 
semblent se fonder sur deux conceptions différentes de la graine. Ainsi, ce qui attire l'attention des élèves, c'est d'abord et surtout «la petite plante» (6, 12, et 34). Quand l'enseignante attire leur attention sur «la peau», ils arrivent bien à voir «le couvercle» (14), la partie «en dedans», c'est encore «la graine» (26), la partie qui fait «les haricots» (21) et d'où sort «la petite plante» (34). Pour l'enseignante, une graine, c'est d'abord «la peau» qui protège «les deux parties» qui, comme elle le dira plus loin, «donnent la nourriture à la petite plante».

L'enseignante semble s'attendre à ce que les élèves décrivent la graine sans que la conception qu'ils s'en font ne vienne donner un sens à leurs observations. Il faudrait plutôt qu'ils la décrivent en adoptant la conception qu'elle s'en fait. Un peu comme si la perception donnait un accès direct aux choses telles qu'elles sont. C'est d'ailleurs l'impression laissée par une autre enseignante de première année quand elle explique à ses élèves: "C'est la peau qui te va te dire si c'est chaud ou si c'est froid" (TXOB01-1025). Plus loin, elle suggère: «Les yeux sont comme deux minuscules caméras qui vont te montrer ce que tu vois" (ТХOB01-1225).

Les entretiens que nous avons eus avec ces deux enseignantes nous permettent de conclure qu'elles souscrivent à l'idéologie empirico-réaliste de la science, tout comme, semble-t-il, une vaste majorité des enseignants (Désautels et Larochelle, 1989). Selon ces deux enseignantes, la science n'aurait que de «bonnes réponses». Ainsi, les connaissances scientifiques décriraient les choses telles qu'elles sont. Ces connaissances découleraient de façon directe de la simple observation des choses et seraient d'une évidence telle qu'on ne saurait les considérer comme étant de nature problématique. Ces connaissances se retrouveraient également dans les livres et seraient transmissibles au moyen de la langue, cette dernière permettant justement de décrire les choses comme elles sont.

Dans l'ensemble, ces enseignantes se disent assez satisfaites des stratégies pédagogiques mises en œuvre dans leur cours de sciences. Ces stratégies sont cohérentes avec leurs conceptions de la science et de l'élève en tant qu'apprenant. Ainsi, même si ces enseignantes réalisent que les élèves ont de nombreuses "idées" (relatives à leurs conceptions), elles ne leur accordent pas pour autant un statut cognitif de constructeur de connaissances. À une question portant sur les «idées» des élèves, une enseignante rétorque: «C'est important parce que ça enrichit. Il y en a [des élèves] qui en savent beaucoup et il y en a qui en savent un peu. C'est tout un enrichissement à cause de ce que les élèves peuvent apporter à la discussion" (EXEN04674). Par contre, selon cette enseignante, c'est plutôt le savoir qu'elle leur «donne» et qu'elle appelle « la base de la science» que les élèves doivent comprendre et sur lequel ils doivent bâtir (EXEN02-429) ${ }^{9}$.

Il faut dire que ces enseignantes sont confortées dans leur perspective par l'orientation pédagogique prédominante en Amérique du Nord qui se fonde sur l'objectivisme et vise essentiellement la transmission de connaissances (Snow, 1990; Tobin, 1993). Cette orientation est d'ailleurs reflétée par de nombreux programmes d'études en sciences. Ces derniers, bien qu'offrant une certaine ouverture sur la science en

9. Ces idées sont approfondies ailleurs (Laplante, 1993, 1996, 1997). 
tant que «moyen de connaître», projettent, par le grand nombre de thèmes exploités et de concepts scientifiques abordés, une image de la science comme étant essentiellement un "ensemble de connaissances» (Cleminson, 1990). En ce sens, les programmes d'études en sciences sont encore loin d'avoir adopté les changements sur le plan du contenu et des stratégies pédagogiques qu'une approche constructiviste entraînerait (Duit et Confrey, 1996). Cette situation n'est pas sans placer les enseignants et les enseignantes devant un dilemme, à savoir s'il faut «couvrir la matière» ou favoriser le développement cognitif des élèves.

\section{Discussion}

\section{Changements et obstacles aux changements en didactique des sciences}

Certains pourraient croire qu'il suffirait de montrer à ces enseignantes comment adopter une approche pédagogique dans laquelle les connaissances abordées en classe ne seraient pas vues comme étant données et non problématiques, mais résulteraient plutôt d'un questionnement des élèves et seraient le fruit de leurs activités d'exploration, de documentation et de synthèse. Une approche dans laquelle tant les connaissances que leurs modes de production feraient l'objet d'une négociation, une approche dans laquelle la langue serait non seulement un moyen de communication et d'interaction sociale, mais aussi un véritable outil de cognition, une approche dans laquelle les élèves seraient perçus comme de véritables constructeurs de connaissances capables d'investigations scientifiques à leur niveau. D'autres voudraient peut-être même qu'on présente à ces enseignantes certains modèles pédagogiques d'orientation constructiviste développés au cours des dernières années.

Il est facile de comprendre que de tels efforts seraient voués à l'échec. D'abord, il faudrait que ces enseignantes se montrent insatisfaites de leur propre modèle pédagogique afin d'être ouvertes à l'idée d'en explorer d'autres. Comme Pépin (1994), ces enseignantes se demanderaient, et avec raison, pourquoi abandonner des savoirs pratiques qui se sont montrés viables jusqu'à maintenant. De plus, il est peu probable que de tels modèles apparaissent intelligibles, plausibles et féconds à leurs yeux. Enfin, la diversité des modèles pédagogiques d'orientation constructiviste proposés nous laisse croire que l'approche à favoriser dans un contexte donné doit nécessairement prendre en compte la situation d'enseignement et d'apprentissage telle qu'elle est vécue par les acteurs qui l'animent. En ce sens, les enseignantes du primaire avec lesquelles nous menons une recherche-action en classe nous laissent savoir que leurs préoccupations immédiates sont plus de nature pédagogique qu'épistémologique. En effet, elles se montrent préoccupées par le manque de matériel et de temps ainsi que par leur propre manque de connaissances scientifiques et pédagogiques. Il ne leur vient pas immédiatement à l'esprit de remettre en question la nature des connaissances scientifiques et des conditions menant à leur production.

Comment faire alors pour amener les changements souhaités dans les pratiques pédagogiques? Selon Tobin (1993, 1995), les modèles d'orientation constructiviste peuvent nous aider à mieux comprendre et agir dans un tel contexte. Ainsi, il faudrait 
d'abord amener les enseignants et enseignantes à réfléchir de façon critique à leur enseignement selon des images, des métaphores, des croyances et des valeurs pour qu'ils en arrivent à reconceptualiser leurs rôles dans la classe. Plus spécifiquement, il faudrait les amener à prendre conscience de leurs conceptions de la science, de l'enseignement, de l'apprentissage, puis à voir comment ces conceptions orientent les stratégies pédagogiques mises en œuvre dans leur classe. Il faudrait même les amener à reconsidérer certains aspects de leur épistémologie personnelle. Ce n'est que dans un tel contexte que de nouveaux modèles pédagogiques d'orientation constructiviste pourraient leur être présentés et faire l'objet d'une expérimentation dans leur classe. Tout en continuant leur enseignement et leur réflexion, en développant de nouvelles images et métaphores décrivant leurs rôles changeants, les enseignants et les enseignantes pourraient en arriver à construire une vision personnelle de l'enseignement et de l'apprentissage dans un contexte constructiviste et prendre les mesures nécessaires pour la réaliser.

De tels changements ne sont réalisables que dans la mesure où ils sont amorcés de l'intérieur de l'école et engagent directement les personnes concernées. Il faudrait que le climat institutionnel leur soit favorable et soutienne les enseignants et les enseignantes dans leur démarche (Tobin, 1995). De plus, ces changements nécessiteraient l'élaboration de nouveaux programmes d'études, de nouvelles stratégies et ressources pédagogiques compatibles avec la thèse constructiviste (Duit, 1995). De tels changements amèneraient une transformation importante de la culture de l'école telle qu'on la connaît (Tobin, 1995). Comme l'affirme Pépin (1994), les enjeux du constructivisme ne se limitent pas à la didactique, ils envahissent tout le champ de l'éducation.

On le constate, l'intégration de la thèse constructiviste aux pratiques éducatives cause plus d'un dilemme aux acteurs œuvrant en didactique des sciences et les défis à relever sont des plus nombreux.

\section{Références bibliographiques}

ARCÀ, M. et CARAVITA, S. (1993). Le constructivisme ne résout pas tous les problèmes. ASTER, 16(1), 78-101.

ASTOLFI, J.-P. et DEVELAY, M. (1989). La didactique des sciences. Paris: Presses universitaires de France.

ASTOLFI, J.-P. et PETERFALVI, B. (1993). Obstacles et construction de situations didactiques en sciences expérimentales. ASTER, 16(1), 103-141.

BAIRD, J. R. et WHITE, R. T. (1996). Metacognitive strategies in the classroom. Dans D. F. Treagust, R. Duit et B. F. Fraser (dir.), Improving Teaching and Learning in Science and Mathematics (p. 190-211). New York: Teachers College Press. 
BEDNARZ, N. et GARNIER, C. (dir.) (1989). Construction des savoirs: obstacles et conflits. Montréal: Cirade et Agences d'Arc.

CLEMINSON, A. (1990). Establishing an epistemological base for science teaching in the light of contemporary notions of the nature of science and of how children learn science. Journal of Research in Science Teaching, 27(5), 429-445.

DÉSAUTELS, J. (1983). Les conceptions spontanées des élèves et l'apprentissage des sciences. Vie pédagogique, 27, 19-23.

DÉSAUTELS, J. et LAROCHELLE, M. (1989). Qu'est-ce que le savoir scientifique? Québec: Presses de l'Université Laval.

DE VECCHI, G. et GIORDAN, A. (1989). L'enseignement scientifique, comment faire pour que «ça marche». Nice: Z'Éditions.

DRIVER, R. (1983). The Pupil as a Scientist? Milton Keynes, UK, Open University Press.

DRIVER, R., GUESNE, E. et TIBERGHIEN, A. (dir.) (1985). Children's Ideas in Science. Milton Keynes, UK: Open University Press.

DUIT, R. (1995). The constructivist view: A fashionable and fruitful paradigm for science education research and practice. Dans L. P. Steffe et J. Gale (dir.), Constructivism in Education (p. 271-285). Hillsdale, NJ : Lawrence Erlbaum Associates.

DUIT, R. et CONFREY, J. (1996). Reorganizing the curriculum and teaching to improve learning in science and mathematics. Dans D. F. Treagust, R. Duit, et B. J. Fraser (dir.), Improving Teaching and Learning in Science and Mathematics (p. 79-93). New York: Teachers College Press.

DUIT, R. et TREAGUST, D. F. (1995). Students' conceptions and constructivist teaching approaches. Dans B. J. Fraser et H. J. Walberg (dir.), Improving science education (p. 46-69). Chicago, IL: National Society for the Study of Education et University of Chicago Press.

FENSHAM, P., GUNSTONE, R. et WHITE, R. (dir.) (1994). The Content of Science. A Constructivist Approach to Its Teaching and Learning. London: Falmer Press.

FOSNOT, C. T. (1996). Constructivism: Theory, Perspectives and Practice. New York: Teachers College Press.

GALLAS, K. (1995). Talking Their Way into Science. New York: Teachers College Press.

GIORDAN, A., GIRAULT, Y. et CLÉMENT, P. (dir.) (1994). Conceptions et connaissances. Berne: Peter Lang.

GIORDAN, A. (dir.) (1983). L'élève et/ou les connaissances scientifiques. Berne: Peter Lang.

HEWSON, P. W. (1981). A conceptual change approach to learning science. European Journal of Science Education, 3(4), 383-396. 
HEWSON, P. W. (1996). Teaching for conceptual change. Dans D. F. Treagust, R. Duit et B. J. Fraser (dir.), Improving Teaching and Learning in Science and Mathematics (p. 131-140). New York: Teachers College Press.

HEWSON, P. W. et HEWSON, M. G. (1984). The role of conceptual conflict in conceptual change and the design of instruction. Instructional Science, 13, $1-13$.

LAPLANTE, B. (1993). Langue et science dans les classes d'immersion: représentations et stratégies pédagogiques d'enseignantes du primaire (thèse de doctorat). Québec: Université Laval.

LAPLANTE, B. (1996). Stratégies pédagogiques et représentation de la langue dans l'enseignement des sciences en immersion française. La Revue canadienne des langues vivantes, 52(3), 440-463.

LAPLANTE, B. (1997). Teachers' beliefs and instructional strategies in science: Pushing analysis further. Science Education, 81(3), 277-294.

LAROCHELLE, M. et BEDNARZ, N. (1994). À propos du constructivisme et de l'éducation. Revue des sciences de l'éducation. XX(1), 5-19.

LAROCHELLE, M. et DÉSAUTELS, J. (1992). Autour de l'idée de science: itinéraires cognitifs d'étudiants et d'étudiantes. Québec: Presses de l'Université Laval.

NUSSBAUM, J. et NOVICK, S. (1982). Alternatives frameworks, conceptual conflict and accommodation: Toward a principled teaching strategy. Instructional Science, 11(3), 183-200.

PÉPIN, Y. (1994). Savoirs pratiques et savoirs scolaires: une représentation constructiviste de l'éducation. Revue des sciences de l'éducation, XX(1), 63-85.

RUEL, F. (1992). À propos du constructivisme. Dans M. Larochelle et J. Désautels (dir.), Autour de l'idée de science: itinéraires cognitifs d'étudiants et d'étudiantes (p. 18-32). Québec: Presses de l'Université Laval.

STEFFE, L. P. et GALE, J.(dir.) (1995). Constructivism in Education, Hillsdale, NJ: Lawrence Erlbaum Associates.

STRIKE, K. A. et POSNER, G. J. (1982). Conceptual change and science teaching. European Journal of Science Education, 4(3), 231-240.

SNOW, M. A. (1990). Instructional methodology in French language immersion. Dans A. M. Padilla, H. H. Fairchild et C.M. Valadez (dir.), Foreign Language Education. Issues and Strategies (p. 156-171). Newbury Park, CA: Sage.

TOBIN, K. (1993). The Practice of Constructivism in Science Education. Hillsdale, NJ: Lawrence Erlbaum Associates.

TOBIN, K. (1995). Teacher change and the assessment of teacher performance. Dans B. J. Fraser et H. J. Walberg (dir.), Improving Science Education (p. 145170). Chicago, IL: National Society for the Study of Education et University of Chicago Press. 
TREAGUST, D. F., DUIT, R. et FRASER, B. J. (dir.) (1996). Improving Teaching and Learning in Science and Mathematics. New York: Teachers College Press.

WANDERSEE, J. H., MINTZES, J. J. et NOVACK, J. D. (1994). Research on alternative conceptions in science. Dans D. L. Gabel (dir.), Handbook of Research on Science Teaching and Learning (p. 177-210). New York: Macmillan. 